\title{
Role of mammalian target of rapamycin signaling in autophagy and the neurodegenerative process using a senescence accelerated mouse-prone 8 model
}

\author{
YANYONG WANG* , QINYING MA* , XIAOWEI MA, ZHONGXIA ZHANG, NA LIU and MINGWEI WANG \\ Department of Neurology, The First Hospital of Hebei Medical University; \\ Laboratory of Brain Aging and Cognitive Neuroscience of Hebei Province, Shijiazhuang, Hebei 050031, P.R. China
}

Received August 24, 2015; Accepted December 23, 2016

DOI: $10.3892 /$ etm.2017.4618

\begin{abstract}
The mammalian target of rapamycin (mTOR) kinase is an inhibitor of autophagy, which is an intracellular system involved in the degradation of long-lived proteins and organelles in lysosomes. Recent evidence suggests that the steady incline in mTOR function during aging may be associated with the cognitive decline related to aging and may also promote development of Tau pathology. At present, the senescence accelerated mouse prone 8 (SAMP8) is an experimental model that has been proposed for the study of age-related neurodegenerative changes associated with aging. In the present study, mTOR signaling in the hippocampus of SAMP8 newborn mice and in the control-strain SAMR1 mice was investigated. Consequently, hyper phosphorylated Tau (pS199 or pS396) and upregulated mTOR activity were observed in SAMP8 when compared with SAMR1; however, $0.5 \mu \mathrm{M}$ rapamycin administration significantly reduced the levels of phosphorylated Tau and p70S6K (pT389) in SAMP8 mice. Related to these findings, SAMP8 exhibited an increase in the neuronal loss of hippocampus that was associated with lower levels of anti-apoptotic proteins. These results indicate that mTOR signaling participates in the neurodegenerative process and rapamycin administration may protect neurons of SAMP8 mice and may have a potential role in curing cognitive decline.
\end{abstract}

Correspondence to: Dr Mingwei Wang, Department of Neurology, The First Hospital of Hebei Medical University; Laboratory of Brain Aging and Cognitive Neuroscience of Hebei Province, 89 Dong-gang Road, Shijiazhuang, Hebei 050031, P.R. China

E-mail: mwwang2007@hotmail.com

*Contributed equally

Key words: mammalian target of rapamycin signaling, autophagy, rapamycin, hippocampus, senescence accelerated mouse-prone 8, aging

\section{Introduction}

The mammalian target of rapamycin (mTOR) has attracted attention due to its critical role in cellular life sustaining activity and vital involvement in a variety of diseases (1). Deregulation of the mTOR signaling pathway affects essential cellular processes and is associated with a number of pathological conditions, including cancer, obesity, type 2 diabetes, and neuron diseases (2). In the neuron system, decreased mTOR signaling is associated with neurodegeneration (3), whereas excess activation of mTOR signaling leads to the abnormal development of neurons and glia, which results in brain malformation (4).

Although mTOR provides positive effects on anabolic processes, growth may be stimulated by negatively regulating autophagy, the central degradative process in cells (2). Autophagy is involved in recycling damaged organelles and is required for the organismal and cellular adaptation to nutrient starvation $(2,5)$. This conserved process aids the maintenance of cellular homeostasis by removing damaged or toxic intrinsic components (6). Furthermore, several studies have suggested that intracellular protein degradation pathways, such as autophagy, are deregulated in neurodegenerative diseases and may possess vital roles in the etiology of neuron-related pathologies (7-9). mTOR signaling is considered to be one of the major pathways involved in the regulation of autophagy and the implications of mTOR signaling in neurodegenerative diseases has been investigated over the last decade (10). Moreover, mTOR negatively regulates the biogenesis of lysosomes, a group of multifunctional organelles that have the capacity to degrade many types of cellular components (11-13).

Rapamycin is a specific inhibitor of mTOR that acts by binding to the FK506-binding protein $12 \mathrm{kDa}$ (FKBP12), forming a molecular complex that impairs mTOR activity $(14,15)$. mTOR kinase inhibitors such as rapamycin are more efficient than the first generation of rapalogs in promoting autophagy and blocking protein synthesis (16), and it has therefore been suggested that mTOR kinase inhibitors may be more effective in treating diseases associated with the formation and accumulation of protein aggregates. Furthermore, rapamycin is able to reduce the rate of protein synthesis and 
thus hinder the aggregation of misfolded proteins. This implies that alternative effectors of mTOR signaling may have roles in the development of neuron-related pathologies (17).

Additionally, the promotion of autophagy linked to mTOR inhibition could mediate some effects of mTOR on longevity. A previous study has indicated that suppression of autophagy gene activity hindered the life span extension in worms with aberrant TOR signaling (18). Therefore, inducing autophagy may reduce the aging process while promoting the breakdown of abnormal proteins and damaged organelles. In a previous report, the Interventions Testing Program (ITP) from the National Institute on Aging demonstrated that rapamycin-induced inhibition of mTOR expanded the life span in mice $(19,20)$.

Therefore, it was hypothesized that the mTOR signaling pathway may possess a key role in the neurodegenerative and aging process of senescence accelerated mouse-prone 8 (SAMP8) mice. Furthermore, treatment with rapamycin and regulating the mTOR pathway may improve the neurodegenerative changes. To test this hypothesis, phosphorylation levels of mTOR and its substrate, p70S6 kinase (p70S6K) was detected. Additionally, the expression levels of anti-apoptotic protein, B cell lymphoma-2 (Bcl-2), were evaluated to investigate the alteration of mTOR signaling pathway in aged SAMP8 mice and primary neurons. As the approach of inducing autophagy has been suggested to aid the removal of abnormal protein aggregates and promote survival in neurons, we investigated whether autophagy may be neuroprotective via preventing cell death in SAMP8 mice.

\section{Materials and methods}

Antibodies and reagent. Mouse monoclonal antibodies (mAbs) for Tau (Tau46) (cat. no. 4019) and phospho-Tau (Ser396) (cat. no. 9632) and rabbit mAbs for beclin-1 (D40C5) (cat. no. 3495), phospho-mTOR (Ser2448) (cat. no. 2971), and phospho-p70 S6 (Thr389) (cat. no. 9205) were purchased from Cell Signaling Technology, Inc. (Danvers, MA, USA). Rabbit polyclonal antibody (pAb) phospho-Tau (Ser199) (cat. no. 44-734G) was obtained from Thermo Fisher Scientific, Inc. (Waltham, MA, USA). Mouse mAb MAP 2a, b, c (Microtubule-Associated Protein) Ab-3 (cat. no. MS-250-P) was from Thermo Fisher Scientific, Inc. Rabbit pAb for LC3B (cat. no. ab48394) was supplied from Abcam (Cambridge, MA, USA). Rabbit pAbs for mTOR (P2476) (cat. no. BS1555) and Bcl-2 (cat. no. BS1511) were purchased from Bioworld Technology, Inc. (St. Louis Park, MN, USA). Infrared-labeled second antibodies (cat.no. 611-731-127) were obtained from LI-COR Biosciences, Inc. (Lincoln, NE, USA). B-27 Serum-free supplement, fetal bovine serum (FBS) and Neurobasal-A medium were supplied from Thermo Fisher Scientific, Inc. CelLytic MT Cell Lysis Reagent was from Sigma-Aldrich (Merck KGaA, Darmstadt, Germany).

Animals. Newborn male SAMP8 and SAMR1 mice were purchased, with six mice in each group. All the mice were maintained and exposed to a 12-h light-dark cycle, had free access to the food and water ad libitum and were kept at a temperature of $20-22^{\circ} \mathrm{C}$. The present study was conducted in accordance with the guidelines established by the Local Animal Care and Use committee.
Primary neuron culture. Primary cultured neurons were established using hippocampus tissues from one to three-day-old SAMP8 and SAMR1 mice. Mice were then sacrificed by decapitation to avoid damaging the brain, and fully immersed in $75 \%$ alcohol for disinfection. An incision was made in the scalp and skull to expose brain and the brain tissue was placed in ice-cold dissection solution. The hippocampus was isolated and dissected free of the meninges and blood vessels under a dissecting microscope. The tissue was diced into small cubes and dissociated by incubation with $0.125 \%$ trypsin for $15-25 \mathrm{~min}$ at $37^{\circ} \mathrm{C}$. Dulbecco's modified Eagle's medium (DMEM), supplemented with $10 \%$ FBS to terminate the digestion, was subsequently added. The tissue was left to precipitate using repeated blows with a polished glass pipette and the supernatant was transferred to a new tube. The supernatant was centrifuged at $114.6 \mathrm{x} \mathrm{g}$ at $16^{\circ} \mathrm{C}$ for $5 \mathrm{~min}$, discarded and the DMEM containing $10 \%$ FBS was added to the precipitation to make a single cell suspension. Cells were seeded in $35-\mathrm{mm}$ dishes $\left(3.37 \times 10^{5} / \mathrm{cm}^{2}\right)$ at $37^{\circ} \mathrm{C}$ in a humidified atmosphere of $5 \% \mathrm{CO}_{2}$. Overnight, the culture medium was replaced with Neurobasal-A medium containing 2\% B27 serum-free medium. The culture medium was changed every three days.

At the seventh day of culturing the cells, neurons from SAMP8 mice were treated with either 0.5 or $1.0 \mu \mathrm{M}$ rapamycin (Sigma-Aldrich; Merck KGaA) and plated in culture medium for three days. The neuron cells were fixed in phosphate-buffered saline (PBS) followed by either immunofluorescence or western-blot analysis. There were four groups in this part of experiment ( $n=6$ per group): Neurons-SAMR1 group; neurons-SAMP8 group; neurons-SAMP8 $+0.5 \mu \mathrm{M}$ rapamycin group; and neurons-SAMP8 $+1.0 \mu \mathrm{M}$ rapamycin group.

Immunocytochemistry. At the tenth day, neurons cultured in dishes were fixed for 30 min with $4 \%$ paraformaldehyde in PBS. Cells were washed three times in PBS and made permeable with $0.5 \%$ Triton $\mathrm{X}-100$ and $5 \%$ goat serum (Invitrogen; Thermo Fisher Scientific, Inc.) in PBS for $40 \mathrm{~min}$, at room temperature. Immunolabeling was performed with mouse mAb MAP 2a, b, c Ab-3 (1:100), at $4^{\circ} \mathrm{C}$ overnight. Cells were washed three times in PBS and incubated with TRITIC-labeled secondary antibody (1:100; cat. no. ZF0313; Beijing Xinqiao Technology Development Co., Ltd., Beijing, China) at $4^{\circ} \mathrm{C}$ for 10 days. Labeled cells were examined using an inverted fluorescence microscope (Nikon Corporation, Tokyo, Japan) with an NIS imaging analysis system (Nikon Corporation). Neurons were indicated by red fluorescence.

Protein extraction. Animals were anesthetized with intraperitoneal injection of $2 \%$ sodium pentobarbital (40-50 mg/kg), then sacrificed by decapitation. The cerebral cortex and hippocampus were dissected, immediately weighed, frozen in liquid nitrogen, and stored at $-80^{\circ} \mathrm{C}$. Protein extracts were prepared in RIPA cell lysis buffer (Thermo Fisher Scientific, Inc.). The lysates were sonicated in an iced water bath for $30 \mathrm{~min}$ and centrifuged at $37,318.4 \mathrm{x}$ g at $4^{\circ} \mathrm{C}$ for $20 \mathrm{~min}$ to collect the supernatants. The protein concentration was measured using a BCA protein assay reagent (Sigma-Aldrich; Merck KGaA) according to manufacturer's instructions. 


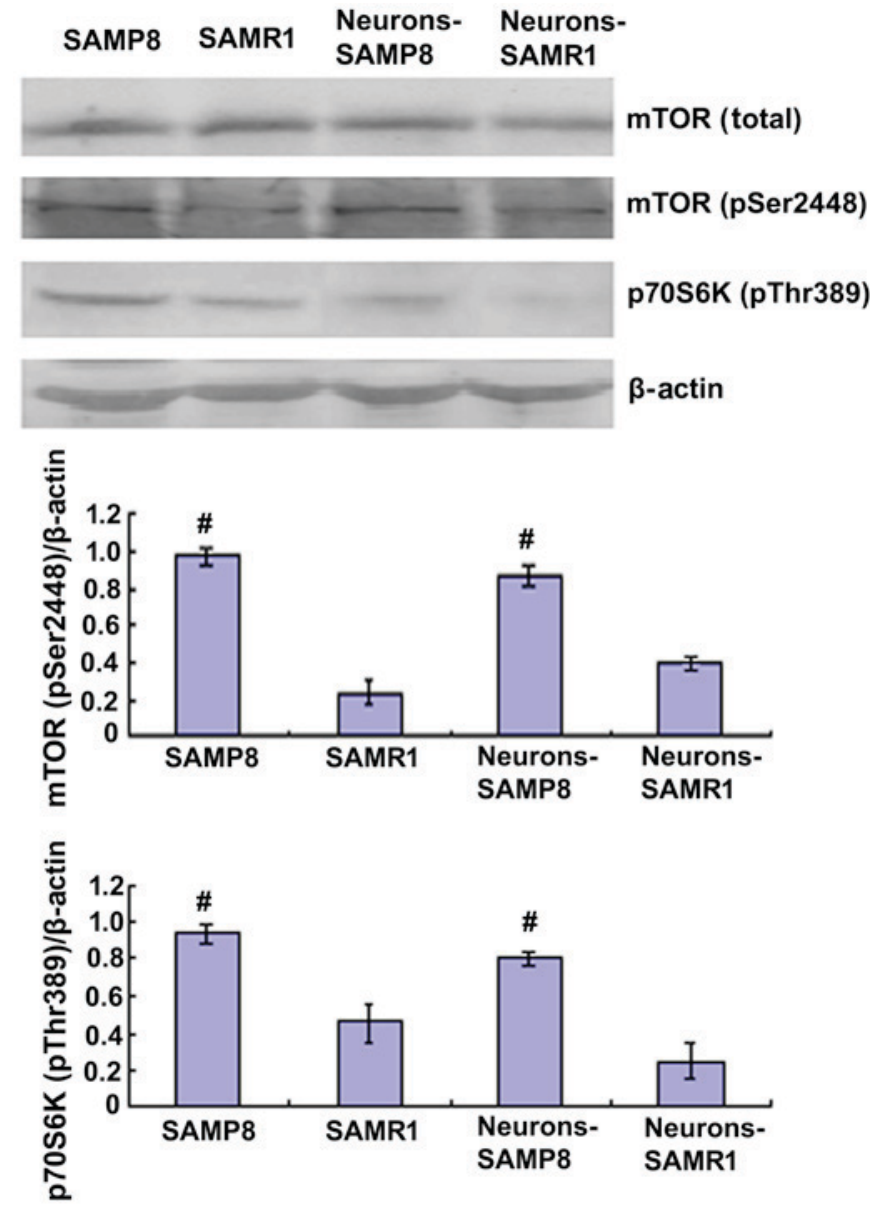

Figure 1. Upregulation of the mTOR pathway in aged SAMP8 compared with SAMR1 in vitro. Western-blot analysis showed the protein expression levels of mTOR (pSer2448) and p70S6K (pThr389) of aged SAMP8 mice and neurons derived from SAMP8 mice were significantly increased when compared with SAMR1 mice. "P $<0.01$ vs. SAMR1 group. mTOR, mammalian target of rapamycin; SAMP8, senescence accelerated mouse prone 8; SAMR1, senescence accelerated mouse resistant 1. SAMP8 group, brain tissue of SAMP8; SAMR1 group, brain tissue of SAMR1; neurons-SAMP8 group, neurons of SAMP8; neurons-SAMR1 group, neurons of SAMR1.

Western blot analysis. Protein extracts (100 $\mu \mathrm{g} / \mathrm{lane})$ were separated using $10 \%$ SDS-PAGE, transferred to polyvinylidene difluoride (PVDF) membranes. Membranes were incubated with 5\% non-fat milk and TBS $(0.1 \%)$ Tween-20 blocking solution for $2 \mathrm{~h}$ at room temperature overnight and then washed with TBS $(0.1 \%)$ Tween-20 blocking solution for $30 \mathrm{~min}$ at room temperature with gentle shaking. Membranes were probed with primary antibodies against LC3B (1:500), beclin 1 (1:500), TAU (pS396) (1:500), TAU (pS199) (1:500), Tau46 (1:1,000), Phospho-mTOR (Ser2448) (1:1,000), mTOR (1:500), p70S6K (pThr389) (1:500), Bcl-2 (1:500) or $\beta$-actin (1:300). All primary antibodies were diluted with blocking solution and incubated overnight at $4^{\circ} \mathrm{C}$. After washing with TBS-0.1\% Tween-20, the PVDF membranes were incubated with fluorescently-labeled secondary antibodies (1:10,000, diluted in blocking solution) for $1 \mathrm{~h}$ at room temperature, and signals were detected with an Odyssey Infrared Imaging System (LI-COR Biosciences, Inc.). Band quantitation of western blots was determined using the image analysis system, Quantity One (Bio-Rad Laboratories, Inc., Hercules, CA, USA). $\beta$-actin protein was used as control. Each experiment was repeated three times with samples from three different mice.

Statistical analysis. Data are expressed as the mean \pm standard deviation. Statistical significance was evaluated by a one-way analysis of variance or via the Non-parametric test. Analyses were conducted using SPSS 13.0 software (SPSS, Inc., Chicago, IL, USA). $\mathrm{P}<0.05$ was considered to indicate a statistically significant difference.

\section{Results}

Upregulation of mTOR signaling in aged SAMP8 compared with SAMR1. In order to determine the changes in MTOR signaling in the aging of SAMP8 mice, western blot analysis was performed to analyze the protein expression levels of total mTOR and mTOR phosphorylation at Ser2448. Western blotting data indicated that the protein expression levels of mTOR (pSer2448) in the SAMP8 group were significantly increased when compared with the control SAMR1 group in vitro $(\mathrm{P}<0.01$; Fig. 1).

The 70-kDa S6 kinase (p70S6K) is a Ser/Thr-directed kinase that has a vital role in cell growth, differentiation, and cycle control (21). mTOR function is typically established via the steady-state level of phosphorylated p70S6K at Thr389, an epitope which is specifically phosphorylated by mTOR; however, mTOR phosphorylation does not consistently rely on this activity $(22,23)$. Western blot analysis revealed that the protein expression levels of phosphorylated p70S6K in the hippocampal primary neurons of SAMP8 mice were significantly increased when compared with the control SAMR1 group, indicating enhanced mTOR activity in aged mice (Fig. 1).

Rapamycin treatment promoted cell morphology and alleviated Tau phosphorylation of neurons from SAMP8. To determine whether or not mTOR signaling had a direct correlation with the neurodegenerative alterations in primary cultured neurons of SAMP8 mice, immunofluorescence staining was used to observe the cell morphology of neurons when cultured at the tenth day or pretreated with rapamycin for three days. Neurons derived from the control SAMR1 group appeared to be normal with slender and smooth projections (Fig. 2Aa) whereas, neurons extracted from SAMP8 were in a poor state, with fragmented or bead-like processes (Fig. 2Ab). When pretreated with $0.5 \mu \mathrm{M}$ rapamycin, a well-known mTOR inhibitor, the neurons from SAMP8 exhibited an improved appearance when compared with the non-treated SAMP8 group, with smooth and slender projections in some of the neurons (Fig. 2Ac). When pretreated with $1.0 \mu \mathrm{M}$ rapamycin, the neurons exhibited a poorer cell state when compared with the untreated SAMP8 group, with most neurons lacking projections (Fig. 2Ad). The present findings suggested that $1.0 \mu \mathrm{M}$ rapamycin may be harmful to neurons, therefore $0.5 \mu \mathrm{M}$ rapamycin was used to pretreat the neurons from SAMP8 in subsequent experiments.

To further determine the effect of rapamycin administration on cell morphology, western blot analysis was performed to investigate the phosphorylation status of Tau, an important microtubule-stabilizing protein in neurons (24). In the 
A

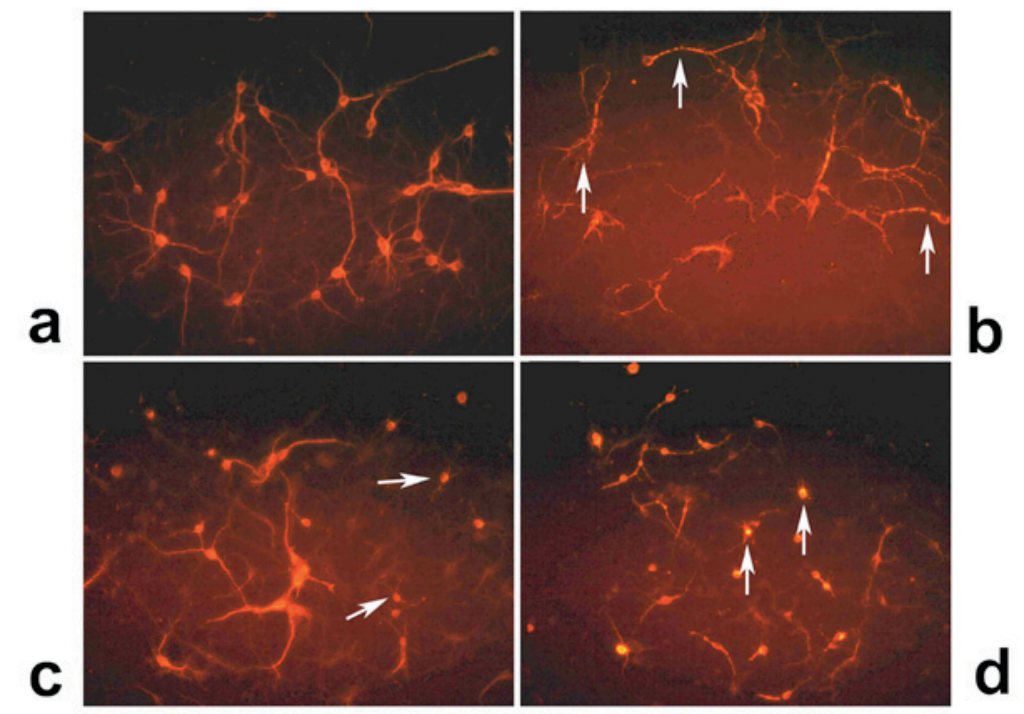

B
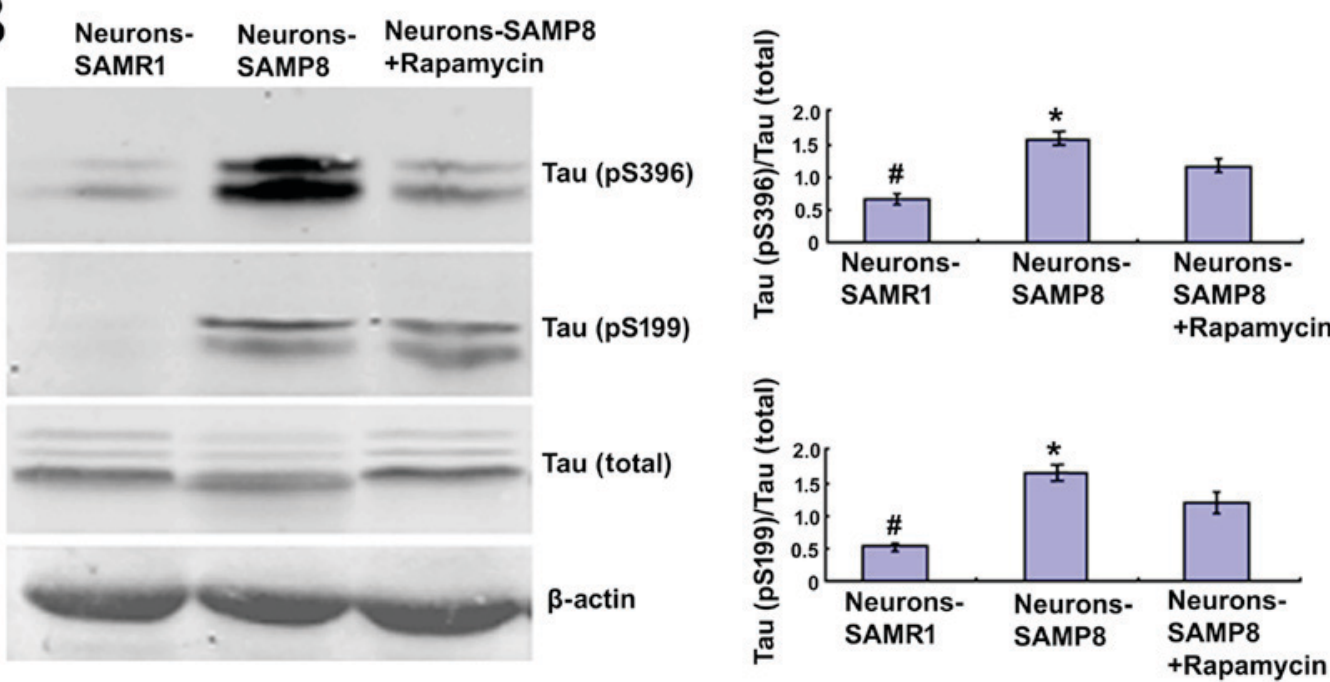

Figure 2. Rapamycin treatment may promote cell morphology and alleviate the Tau phosphorylation of neurons from SAMP8. (A) Cell morphology (magnification, $\mathrm{x} 200$ ). The neurons derived from SAMR1 appear to be in a normal state (a, Neurons-SAMR1). The neurons extracted from SAMP8 were in poor state with most processes broken and exhibited 'bead-like' changes (indicated by the white arrows) (b, Neurons-SAMP8). When pretreated with $0.5 \mu$ M rapamycin, neurons from SAMP8 were partially improved with some smooth and slender processes (indicated by the white arrows; $\mathrm{c}$, Neurons-SAMP8 $+0.5 \mu \mathrm{M}$ rapamycin). When pretreated with $1.0 \mu \mathrm{M}$ rapamycin, the cell state was worse than the untreated group and most neurons lacked projections $(\mathrm{d}, \mathrm{Neurons}-\mathrm{SAMP} 8+1.0 \mu \mathrm{M}$ rapamycin). (B) Western blot analysis was used to investigate Tau phosphorylation. In the neurons-SAMP8 group, the protein expression levels of Tau (pS199) and Tau (pS396) were significantly increased when compared with the control neurons-SAMR1 group. When pretreated with $0.5 \mu \mathrm{M}$ rapamycin for three days, Tau (pS199) and Tau (pS396) exhibited significantly decreased levels of protein expression when compared with the neurons-SAMP8 group. ${ }^{\text {"P }}<0.05$ vs. neurons-SAMP8 + Rapamycin group; ${ }^{\#} \mathrm{P}<0.01$ vs. neurons-SAMP8 group. SAMP8, senescence accelerated mouse prone 8; SAMR1, senescence accelerated mouse resistant 1. SAMP8 group, brain tissue of SAMP8; SAMR1 group, brain tissue of SAMR1; neurons-SAMP8 group, neurons of SAMP8; neurons-SAMR1 group, neurons of SAMR1.

neurons-SAMP8 group, the protein expression levels of Tau $(\mathrm{pS} 199)$ and Tau (pS396) were significantly increased when compared with the control neurons-SAMR1 group $(\mathrm{P}<0.01)$. When pretreated with $0.5 \mu \mathrm{M}$ rapamycin for three days, Tau (pS199) and Tau (pS396) protein expression levels were significantly decreased when compared with the neurons-SAMP8 group $(\mathrm{P}<0.05$; Fig. $2 \mathrm{~B})$. These results suggested that rapamycin treatment may downregulate the phosphorylation of Tau protein and protect the neurons in SAMP8 against degeneration.

Rapamycin may inhibit mTOR signaling and promote autophagy. Rapamycin $(0.5 \mu \mathrm{M})$ was used to pretreat primary neurons extracted from SAMP8. Rapamycin significantly increased the protein expression levels of LC3-II and beclin 1 in the SAMP8 neurons $(\mathrm{P}<0.05$; Fig. 3A). The level of LC3-II is an indicator for the extent of autophagy (25). Therefore, this result indicated that rapamycin may stimulate autophagic activity. Although the LC3-II protein expression levels exhibited in the neurons-SAMR1 group was not significantly different from the neurons in the-SAMP8 group ( $P>0.05)$, the protein expression levels of beclin 1, a well-known key regulator of autophagy (26), were significantly decreased in the neurons-SAMP8 group compared with the neurons-SAMR1 group $(\mathrm{P}<0.05$; Fig. $3 \mathrm{~A})$. This result indicated that the activity of autophagy was lower in the neurons-SAMP8 at the tenth day (Fig. 3A). 

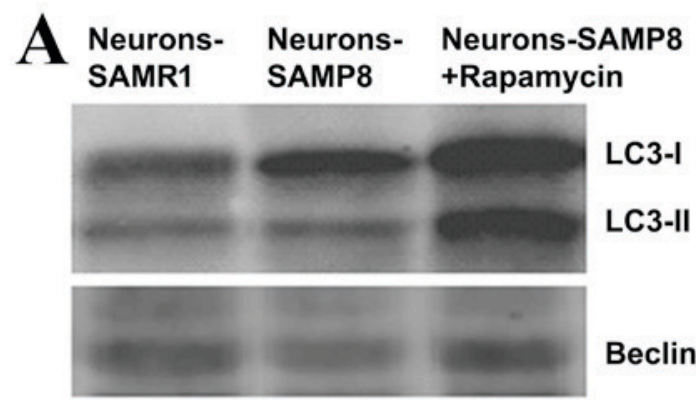

Beclin 1

$\beta$-actin

B

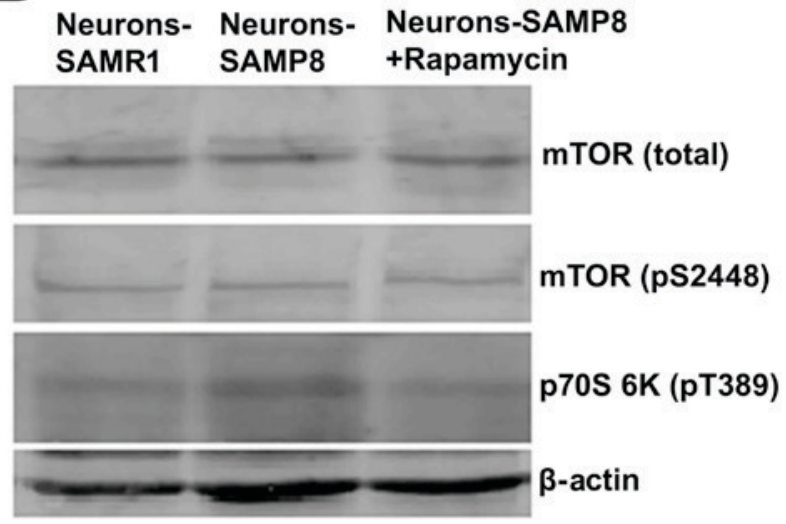

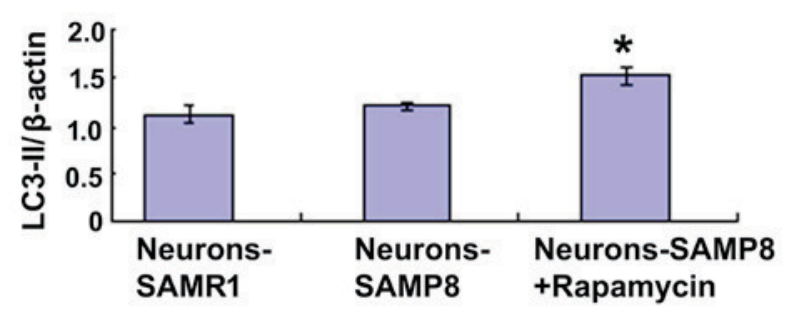
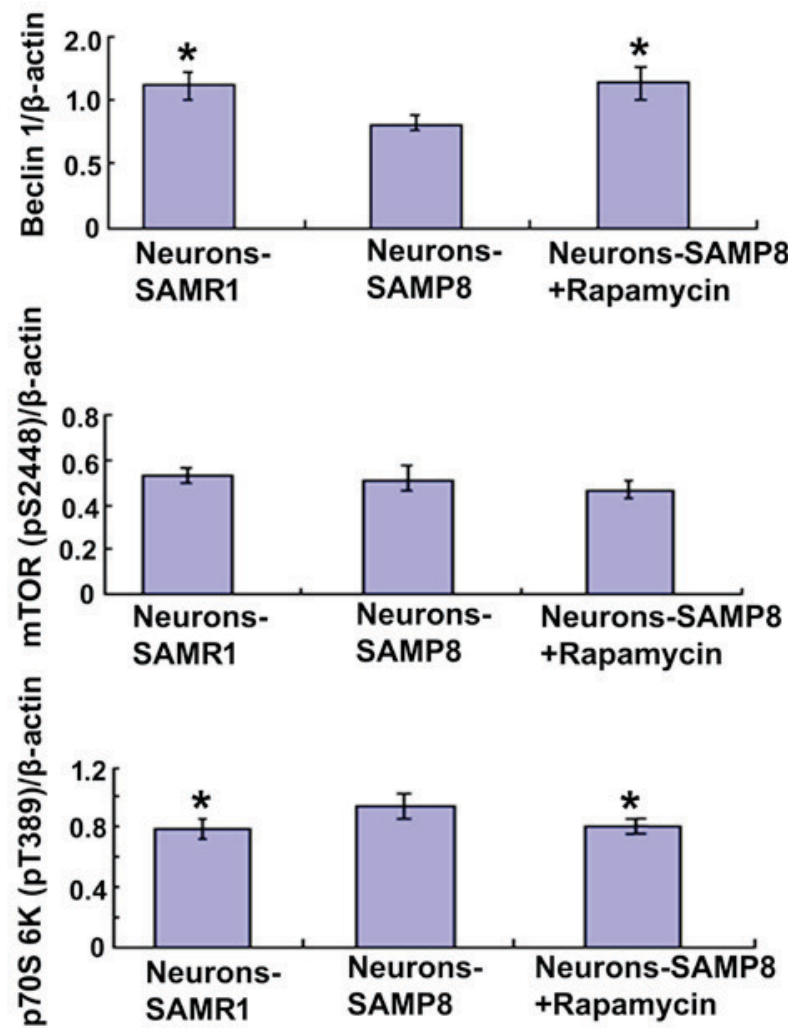

Figure 3. Rapamycin promoted autophagy by inhibiting mTOR signaling. (A) Rapamycin (0.5 $\mu \mathrm{M})$ administration was able to significantly increase the protein expression levels of LC3-II and beclin 1 (both $\mathrm{P}<0.05$ ), suggesting that rapamycin may stimulate autophagic activity. (B) There were no differences in total mTOR and phospho-mTOR $(\mathrm{P}>0.05)$ protein expression levels amongst the three groups; however, the levels of phosphorylated p70S6K at Thr389 were significantly reduced in the rapamycin-pretreated group $(\mathrm{P}<0.05)$, which suggests that mTOR phosphorylation was not always in accordance with its activity. ${ }^{*} \mathrm{P}<0.05$ vs. neurons-SAMP8 group. SAMP8, senescence accelerated mouse prone 8; SAMR1, senescence accelerated mouse resistant 1 ; LC3, microtubule-associated protein 1A/1B-light chain 3; mTOR, mammalian target of rapamycin; neurons-SAMP8 group, neurons of SAMP8; neurons-SAMR1 group, neurons of SAMR1.

The effect of $0.5 \mu \mathrm{M}$ rapamycin on mTOR signaling was determined using western blot analysis in primary neurons. Rapamycin exhibited no effect on the protein expression levels of total mTOR $(\mathrm{P}>0.05)$ and phospho-mTOR $(\mathrm{P}>0.05)$; however, the protein expression levels of phosphorylated p70S6K at Thr389 were significantly decreased in the rapamycin pretreated SAMP8 group when compared with the neurons-SAMP8 group ( $\mathrm{P}<0.05$; Fig. $3 \mathrm{~B}$ ) which suggested that mTOR phosphorylation is not always in accordance with its activity.

Rapamycin decreased the protein expression level of Bcl-2 in primary neurons. Bcl-2 is an anti-apoptotic protein that is able to inhibit macroautophagy (27). The present findings indicated that $\mathrm{Bcl}-2$ protein expression levels in the cortex and hippocampus of 12-month-old SAMP8 mice exhibited no difference when compared with matched SAMR1 mice and the primary neurons from the two strains ( $P>0.05$; Fig. 4A).
However, in the neurons-SAMP8 + rapamycin group, Bcl-2 protein expression levels were significantly decreased when compared with the neurons-SAMP8 group ( $\mathrm{P}<0.05$; Fig. 4B).

\section{Discussion}

The major findings of the present study indicated that the levels of mTOR (pSer2448) and phosphorylated p70S6K at Thr389 were significantly increased in SAMP8 hippocampal neurons when compared with the control SAMR1 group. Furthermore, mTOR activity signaling was upregulated in the neurons of SAMP8. The increase of mTOR signaling in the hippocampus and primary neurons of aged SAMP8 mice may contribute to neurodegenerative disorders in SAMP8.

Rapamycin is a specific inhibitor of mTOR, which has been used in vitro and in vivo to block mTOR function (28). The present results demonstrated that rapamycin administration significantly increased the protein expression levels 
of phosphorylated p70S6K in the SAMP8 group when compared with the SAMR1 group. When treated with $0.5 \mu \mathrm{M}$ rapamycin for three days, the appearance of neurons from the neurons-SAMP8 group were improved and Tau (pS199) and Tau (pS396) protein expression levels were decreased when compared with the untreated neurons-SAMP8 group. Western blot analysis of brain lysates from rapamycin-treated and the control SAMR1 groups showed a significant decrease in the phosphorylation of p70S6K, demonstrating the effective inhibition of rapamycin on the mTOR pathway. Previous data from studies conducted on Alzheimer's disease brains have further strengthened the link of mTOR and Tau and demonstrated that mTOR signaling is selectively increased in neurons predicted to develop neurofibrillary tangles and such an increase correlated with Tau phosphorylation $(21,29,30)$. The present results suggest that rapamycin may provide neuron protection through the inhibition of mTOR and alleviation of Tau phosphorylation.

The autophagy process recycles unnecessary or damaged material, therefore, not only providing nutrients to maintain vital cellular functions in times of starvation but also eliminating potentially harmful cellular material (31). Autophagy is constitutively active in healthy neurons $(32,33)$. A previous study using neuron-specific autophagy protein-deficient mice $\left(\right.$ atg $5^{-/}$or $\operatorname{atg} 7^{-/}$) exhibited abnormal protein aggregates, neurodegeneration and subsequent motor dysfunction, which suggested that autophagy is essential for neuronal homeostasis and quality control (34). Furthermore, a previous study demonstrated a correlation with the cognitive decline in the hippocampal neurons of 12-month-old SAMP8 mice with an increase in ubiquitinated proteins (35). mTOR is able to control protein turnover though inhibiting autophagy (36); therefore, the basal and optimal levels of autophagy are essential for neurons to efficiently remove damaged organelles and misfolded proteins.

Bcl-2 is an anti-apoptotic protein that is able to inhibit autophagy (37). In the present study, when rapamycin was administered, the $\mathrm{Bcl}-2$ protein expression levels declined significantly in the neurons-SAMP8 group, indicating that Bcl-2 may be regulated by rapamycin through mTOR signaling. However, no difference was indicated in the Bcl-2 protein expression levels in the cortex and hippocampus of 12-month-old SAMP8 mice when compared with the matched SAMR1 mice and the primary neurons from the two strains, indicating that Bcl-2 may not participate in the aging process of SAMP8. Results from previous studies have demonstrated that beclin 1 is an important component of the phosphoinositide 3-kinase complex that regulates autophagosome maturation, endocytic trafficking and the downregulation of beclin 1 decreases the activity of autophagy $(37,38)$. Further studies have revealed that starvation induces Jun N-terminal kinase 1 activity, which may phosphorylate Bcl-2, thereby disrupting the association between beclin 1 and $\mathrm{Bcl}-2$ to induce autophagy (39). In an alternative study (40), LC3-II and beclin 1 expression levels were increased in brain areas of seven-month-old SAMP8; At 12 months, LC3-II expression remained increased whereas beclin 1 expression was diminished, suggesting that autophagic activity may increase reactively at the beginning of Alzheimer's disease and decline with aging. The pathological changes of 12-month-old SAMP8 are similar to late-onset Alzheimer's disease from the perspective of autophagy (41).
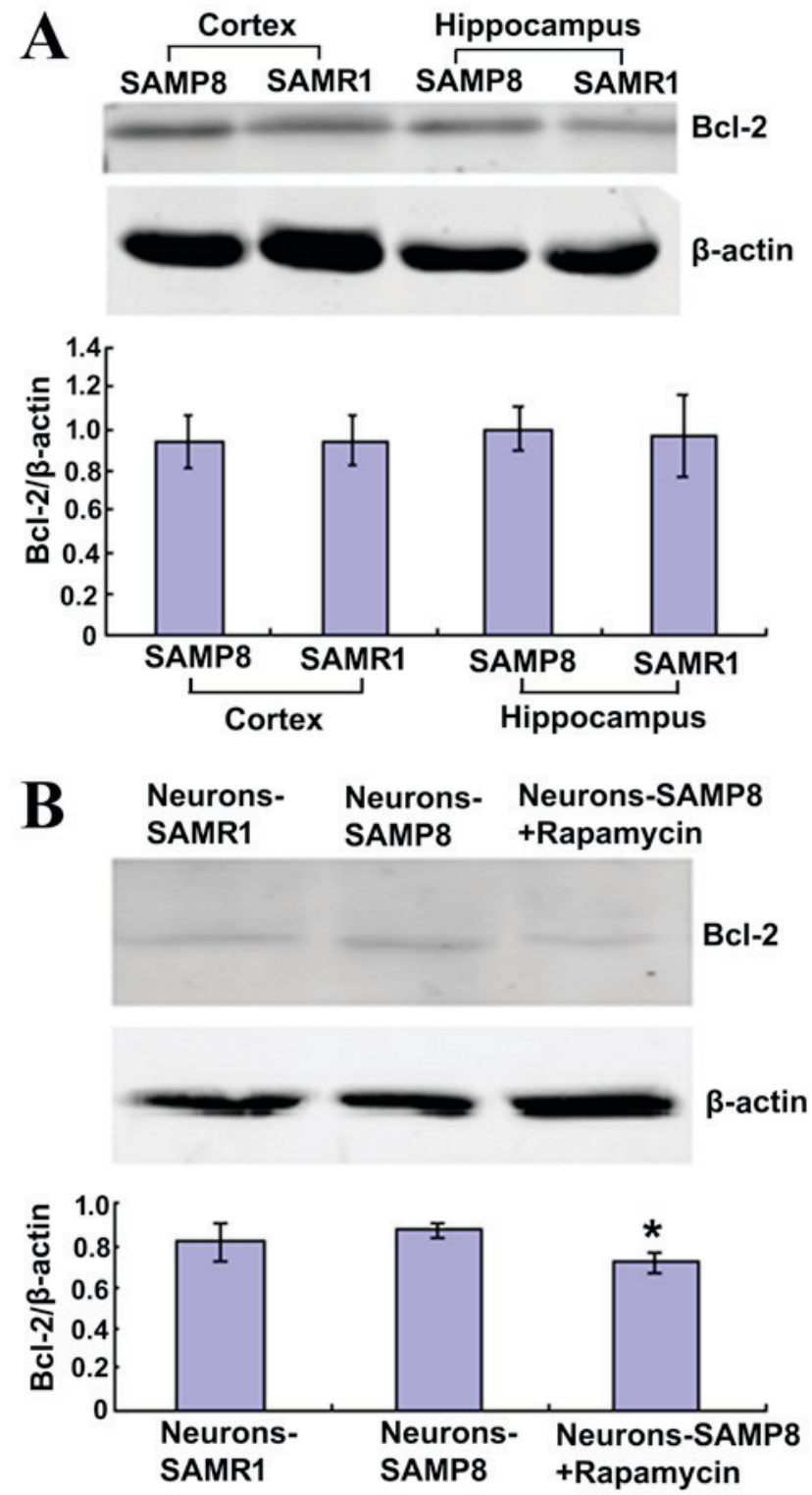

Figure 4. Rapamycin reduced the protein expression levels of Bcl-2 in primary neurons. (A) Protein expression levels of Bcl-2 were similar in the cortex and hippocampus of the two mouse strains. (B) When neurons-SAMP8 were administered $0.5 \mu \mathrm{M}$ rapamycin, the Bcl-2 protein expression levels declined significantly. "P<0.05 vs. neurons-SAMP8 group. SAMP8, senescence accelerated mouse prone 8; SAMR1, senescence accelerated mouse resistant 1; Bcl-2, B cell lymphoma-2; neurons-SAMP8 group, neurons of SAMP8; neurons-SAMR1 group, neurons of SAMR1.

In conclusion, the present study demonstrated that mTOR signaling plays a key role in the neurodegenerative process, and rapamycin administration could protect neurons and alleviate Tau phosphorylation of SAMP8 mice. In addition, rapamycin might have a potential role in reducing cognitive decline. However, the current study of rapamycin was limited to in vitro results. Thus, further in vivo studies in animal models are required.

\section{Acknowledgements}

The present study was supported by the Hebei Department of Health (Grant No ZL20140075; Hebei, China). The authors acknowledge the expert technical assistance of Dr Lingling 
Jiang, and the donation of SAMP8 and SAMR1 mice from Professor David Yew.

\section{References}

1. Keith CT and Schreiber SL: PIK-related kinases: DNA repair, recombination, and cell cycle checkpoints. Science 270: 50-51, 1995.

2. Levine B and Kroemer G: Autophagy in the pathogenesis of disease. Cell 132: 27-42, 2008

3. Takei $\mathrm{N}$ and Nawa $\mathrm{H}$ : mTOR signaling and its roles in normal and abnormal brain development. Front Mol Neurosci 7: 28, 2014.

4. Meijer AJ and Codogno P: Regulation and role of autophagy in mammalian cells. Int J Biochem Cell Biol 36: 2445-2462, 2004.

5. Sarkar S: Regulation of autophagy by mTOR-dependent and mTOR-independent pathways: Autophagy dysfunction in neurodegenerative diseases and therapeutic application of autophagy enhancers. Biochem Soc Trans 41: 1103-1130, 2013.

6. Mariño G, Madeo F and Kroemer G: Autophagy for tissue homeostasis and neuroprotection. Curr Opin Cell Biol 23 198-206, 2011.

7. Rubinsztein DC: The roles of intracellular protein-degradation pathways in neurodegeneration. Nature 443: 780-786, 2006.

8. Pan T, Kondo S, Le W and Jankovic J: The role of autophagy-lysosome pathway in neurodegeneration associated with Parkinson's disease. Brain 131: 1969-1978, 2008.

9. Rivero-Ríos P, Madero-Pérez J, Fernández B and Hilfiker S: Targeting the Autophagy/Lysosomal Degradation Pathway in Parkinson's Disease. Curr Neuropharmacol 14: 238-249, 2016.

10. Wong M: Mammalian target of rapamycin (mTOR) pathways in neurological diseases. Biomed J 36: 40-50, 2013.

11. Ganley IG, Lam du H, Wang J, Ding X, Chen S and Jiang X: ULK1.ATG13.FIP200 complex mediates mTOR signaling and is essential for autophagy. J Biol Chem 284: 12297-12305, 2009.

12. Hosokawa N, Hara T, Kaizuka T, Kishi C, Takamura A, Miura Y, Iemura S, Natsume T, Takehana K, Yamada N, et al: Nutrient-dependent mTORC1 association with the ULK1-Atg13-FIP200 complex required for autophagy. Mol Biol Cell 20: 1981-1991, 2009.

13. Jung CH, Jun CB, Ro SH, Kim YM, Otto NM, Cao J, Kundu M and Kim DH: ULK-Atg13-FIP200 complexes mediate mTOR signaling to the autophagy machinery. Mol Biol Cell 20: 1992-2003, 2009.

14. Chen J, Zheng XF, Brown EJ and Schreiber SL: Identification of an 11-kDa FKBP12-rapamycin-binding domain within the 289-kDa FKBP12-rapamycin-associated protein and characterization of a critical serine residue. Proc Natl Acad Sci USA 92: 4947-4951, 1995.

15. Choi J, Chen J, Schreiber SL and Clardy J: Structure of the FKBP12-rapamycin complex interacting with the binding domain of human FRAP. Science 273: 239-242, 1996.

16. Laplante M and Sabatini DM: mTOR signaling in growth control and disease. Cell 149: 274-293, 2012.

17. King MA, Hands S, Hafiz F, Mizushima N, Tolkovsky AM and Wyttenbach A: Rapamycin inhibits polyglutamine aggregation independently of autophagy by reducing protein synthesis. Mol Pharmacol 73: 1052-1063, 2008.

18. Tóth ML, Sigmond T, Borsos E, Barna J, Erdélyi P, Takács-Vellai K, Orosz L, Kovács AL, Csikós G, Sass M and Vellai T: Longevity pathways converge on autophagy genes to regulate life span in Caenorhabditis elegans. Autophagy 4: 330-338, 2008

19. Harrison DE, Strong R, Sharp ZD, Nelson JF, Astle CM, Flurkey K, Nadon NL, Wilkinson JE, Frenkel K, Carter CS, et al: Rapamycin fed late in life extends lifespan in genetically heterogeneous mice. Nature 460: 392-395, 2009.

20. Miller RA, Harrison DE, Astle CM, Baur JA, Boyd AR, de Cabo R, Fernandez E, Flurkey K, Javors MA, Nelson JF, et al: Rapamycin, but not resveratrol or simvastatin, extends life span of genetically heterogeneous mice. J Gerontol A Biol Sci Med Sci 66: 191-201, 2011.
21. Pei JJ, Björkdahl C, Zhang H, Zhou X and Winblad B: p70 S6 kinase and tau in Alzheimer's disease. J Alzheimers Dis 14: 385-392, 2008

22. Das F, Ghosh-Choudhury N, Mahimainathan L, Venkatesan B, Feliers D, Riley DJ, Kasinath BS and Choudhury GG: Raptor-rictor axis in TGFbeta-induced protein synthesis. Cell Signal 20: 409-423, 2008.

23. Hay N and Sonenberg N: Upstream and downstream of mTOR. Genes Dev 18: 1926-1945, 2004.

24. Meske V, Albert F and Ohm TG: Coupling of mammalian target of rapamycin with phosphoinositide 3-kinase signaling pathway regulates protein phosphatase $2 \mathrm{~A}$ - and glycogen synthase kinase-3-dependent phosphorylation of Tau. J Biol Chem 283: 100-109, 2008

25. Barth S, Glick D and Macleod KF: Autophagy: Assays and artifacts. J Pathol 221: 117-124, 2010.

26. Zhang W, Li Q, Song C and Lao L: Knockdown of autophagy-related protein 6 , Beclin-1, decreases cell growth, invasion, and metastasis and has a positive effect on chemotherapy-induced cytotoxicity in osteosarcoma cells. Tumour Biol 36: 2531-2539, 2015.

27. Pattingre S, Tassa A, Qu X, Garuti R, Liang XH, Mizushima N, Packer M, Schneider MD and Levine B: Bcl-2 antiapoptotic proteins inhibit Beclin 1-dependent autophagy. Cell 122: 927-939, 2005.

28. Dancey JE: Inhibitors of the mammalian target of rapamycin. Expert Opin Investig Drugs 14: 313-328, 2005.

29. An WL, Cowburn RF, Li L, Braak H, AlafuzoffI, Iqbal K, Iqbal IG Winblad B and Pei JJ: Up-regulation of phosphorylated/activated p70 S6 kinase and its relationship to neurofibrillary pathology in Alzheimer's disease. Am J Pathol 163: 591-607, 2003.

30. Pei JJ and Hugon J: mTOR-dependent signalling in Alzheimer's disease. J Cell Mol Med 12: 2525-2532, 2008.

31. Kroemer G, Mariño G and Levine B: Autophagy and the integrated stress response. Mol Cell 40: 280-293, 2010.

32. Lee JA: Neuronal autophagy: A housekeeper or a fighter in neuronal cell survival? Exp Neurobiol 21: 1-8, 2012.

33. Xilouri M and Stefanis L: Autophagy in the central nervous system: Implications for neurodegenerative disorders. CNS Neurol Disord Drug Targets 9: 701-719, 2010.

34. Mizushima N and Levine B: Autophagy in mammalian development and differentiation. Nat Cell Biol 12: 823-830, 2010.

35. Ma Q, Qiang J, Gu P, Wang Y, Geng Y and Wang M: Age-related autophagy alterations in the brain of senescence accelerated mouse prone 8 (SAMP8) mice. Exp Gerontol 46: 533-541, 2011.

36. Díaz-Troya S, Pérez-Pérez ME, Florencio FJ and Crespo JL: The role of TOR in autophagy regulation from yeast to plants and mammals. Autophagy 4: 851-865, 2008.

37. Klionsky DJ, Codogno P, Cuervo AM, Deretic V, Elazar Z, Fueyo-Margareto J, Gewirtz DA, Kroemer G, Levine B, Mizushima N, et al: A comprehensive glossary of autophagy-related molecules and processes. Autophagy 6: 438-448, 2010

38. Klionsky DJ, Abdalla FC, Abeliovich H, Abraham RT, Acevedo-Arozena A, Adeli K, Agholme L, Agnello M, Agostinis P, Diwan A, et al: Guidelines for the use and interpretation of assays for monitoring autophagy. Autophagy 8: 445-544, 2012.

39. Wei Y, Pattingre S, Sinha S, Bassik M and Levine B: JNK1-mediated phosphorylation of $\mathrm{Bcl}-2$ regulates starvation-induced autophagy. Mol Cell 30: 678-688, 2008.

40. Mizushima N and Yoshimori T: How to interpret LC3 immunoblotting. Autophagy 3: 542-545, 2007.

41. Menardo J, Tang Y, Ladrech S, Lenoir M, Casas F, Michel C, Bourien J, Ruel J, Rebillard G, Maurice T, et al: Oxidative stress, inflammation, and autophagic stress as the key mechanisms of premature age-related hearing loss in SAMP8 mouse Cochlea. Antioxid Redox Signal 16: 263-274, 2012. 